\title{
[Reseña de libro] \\ Diagnóstico del funcionamiento de los Consejos Técnicos Regionales de Educación Básica en el marco de un Modelo de Gestión Regional, Hidalgo, 2017
}

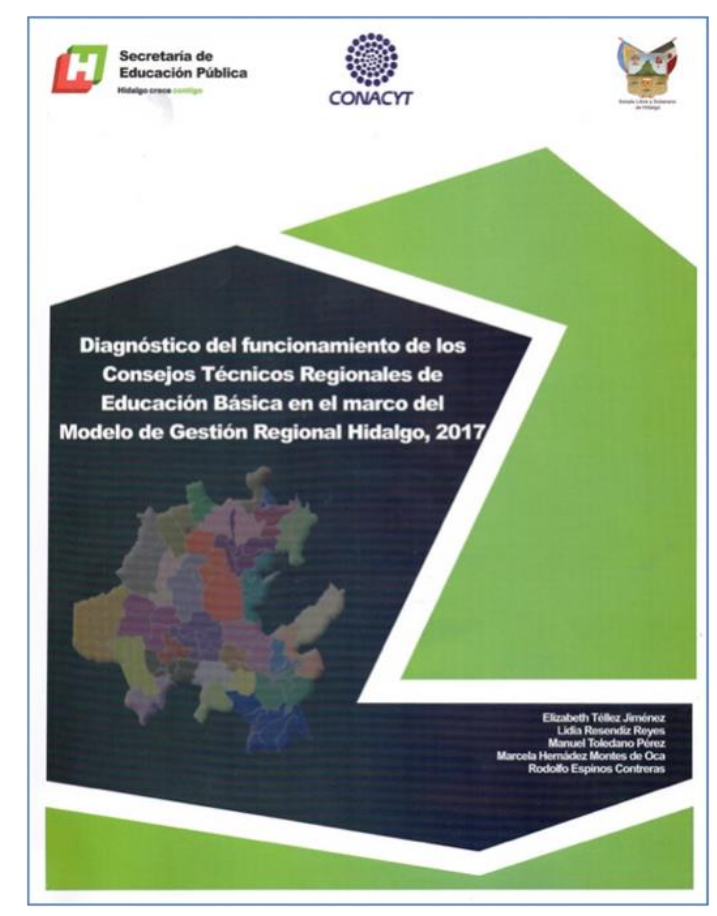

Manuel Toledano Pérez ${ }^{1}$

El diagnóstico es resultado de una investigación educativa, que en su primera etapa estudia el funcionamiento de los Consejos Técnicos Regionales (CTR) de educación básica en el marco del Modelo de Gestión

\footnotetext{
1 Manuel Toledano Pérez. Dirección de Investigación Educativa de la Dirección de Desarrollo Curricular, Secretaría de Educación Pública, Hidalgo, México. Ha realizado investigación educativa acerca de la formación y práctica profesional del docente de historia en educación secundaria. Correo electrónico: manuel t p@yahoo.com.mx

ID: http://orcid.org/0000-0002-2172-7896
} 
Regional, Hidalgo (MGRH), que permitirá en la segunda etapa el diseño de una propuesta para fortalecer la función supervisora y gestión de los CTR. Esta investigación educativa estuvo financiada por el Consejo de Ciencia y Tecnología (CONACyT) y además fortalece la estrategia de operación de los CTR, los Consejos Técnicos de Zona y los Consejos Técnicos de Escolares de Educación Básica y Educación Media Superior para el ciclo escolar 20172018.

La pregunta que orienta el proceso de indagación, es ¿Cómo ha sido el proceso de implementación de los CTR de educación básica en el marco del MGRH y su contribución al desempeño profesional del supervisor escolar y al logro eficaz de la gestión planteada por la reforma educativa vigente?

Construir un Diagnóstico confiable y objetivo, exigió contar con información de primera mano, es decir, directamente de los sujetos que conforman los CTR a fin de reconocer las fortalezas y áreas de oportunidad.

El texto está organizado en siete apartados, El Modelo de Gestión Regional Hidalgo, Proceso metodológico, Aspectos socioeconómicos del estado de Hidalgo, Infraestructura, Población, Estrategias de operación de los Consejos Técnicos Regionales, y Conclusiones.

Contar con el presente Diagnóstico de la implementación de los CTR en el marco del MGRH, 2017, es una gran oportunidad para acceder a información relevante y contextualizada sobre un sector importante en la escena educativa en el estado de Hidalgo, es decir, los supervisores escolares y la cultura colegiada que han venido instalando y desarrollando, con la convicción de que no es posible transitar a mejores escenarios sino se cuenta con un reconocimiento real de lo que ya existe.

\section{El Modelo de Gestión Regional Hidalgo}

Este Modelo plantea una propuesta de transformación institucional del Sistema Educativo del Estado de Hidalgo, que para la elaboración de este Modelo implicó un reconocimiento de las formas y particularidades en las que se había venido ejerciendo la función supervisora en la entidad por los jefes de sector y los supervisores, son figuras directivas que representan la columna vertebral del sistema educativo nacional y estatal ya que a través de ellos circula la información, las reformas que direccionan a la educación y que por lo tanto requieren apoyos no sólo materiales sino también académicos para realizar sus diversas funciones. 


\section{Breve semblanza}

El MGRH se entiende como un sistema en el cual están organizados, articulados y coordinados sus distintos elementos: Componentes, postulados, estructuras y herramientas.

Establece cuatro postulados Territorio regional y escuela, Enfoque regional educativo para el desarrollo sustentable y la sustentabilidad, Democracia social y participativa y el Enfoque estratégico: Pensamiento holístico, Pensamiento sistémico, Pensamiento estratégico que son la base filosófica para organizar el trabajo educativo en la entidad, determinan la base de la estructura organizativa, basados planteados por la planeación estratégica, con una mirada centrada en las escuelas y en el trabajo el aula, la finalidad es fomentar el logro adecuado de los aprendizajes de los alumnos en un marco de calidad, acordes a las exigencias del sistema educativo nacional y a los estándares internacionales de educación básica.

El MGRH establece los principios de: Autonomía responsable, Flexibilidad en las prácticas, Trabajo colaborativo, Corresponsabilidad por los resultados, Rendición de cuentas y Planeación estratégica y flexible, que guian la actuación de los participantes en los CTR, es un determinado modo de concebir y de asumir el quehacer profesional, es un hacer con sentido.

\section{Objetivo y estructura organizativa}

El MGRH se plantea como objetivo general: Contribuir, de manera eficiente, eficaz y pertinente, al mejoramiento continuo y sostenido de la calidad con equidad de los aprendizajes de todos los alumnos de educación básica, mediante el fortalecimiento de la gestión institucional en las 30 regiones educativas del estado de Hidalgo, impulsando estrategias diferenciadas en concordancia con las necesidades educativas y contextuales de cada uno de los centros educativos de la entidad (SEPH, 2014:60).

La estructura organizativa del Modelo de Gestión Regional Hidalgo, queda establecida a partir de los siguientes órganos colegiados: El Comité Técnico Estatal de Educación Básica, El Consejo Técnico Regional, El Consejo Técnico de Zona y El Consejo Técnico Escolar.

\section{Los actores educativos}

Los equipos de supervisión son todo el personal de supervisión escolar (Jefes de sector, supervisores generales, supervisores de zona, jefes de enseñanza) estos equipos representan el principal actor del MGRH ya que se busca propiciar una vinculación real entre los niveles de educación básica y las modalidades educativas, atendiendo tanto las demandas educativas que son comunes de las zonas escolares como las particulares y específicas 
de cada una de las escuelas que tienen a su cargo, teniendo como base, las problemáticas detectadas en las rutas de mejora de los consejos técnicos escolares, identificando y priorizando las que pueden solventar en la zona escolar y gestionando aquellas que requieren de los servicios de apoyo del CTR.

\section{Proceso metodológico}

La mirada metodológica fue de corte cuantitativa para la construcción del Diagnóstico, que para su elaboración se apoyó de la información derivada de la "Encuesta sobre el funcionamiento de los CTR en Hidalgo 2017", cuyo propósito fue identificar y reconocer el funcionamiento de los 30 consejos constituidos en el estado de Hidalgo. Para tal fin, resultó fundamental la obtención de datos primarios que "son aquellos generados por el investigador con el propósito específico de la investigación que se está realizando" (Arriaza, B. 2006:20).

Para obtener la información de cada uno de los componentes, el equipo de trabajo decidió diseñar tres cuestionarios: 1 . Infraestructura de los CTR en Hidalgo 2017, 2. Población de los CTR en Hidalgo 2017 y 3. Estrategias de operación de los CTR en Hidalgo 2016; con una orientación cuantitativa sobre cada uno de los aspectos ya señalados, dando origen a la Encuesta sobre el funcionamiento de los CTR en Hidalgo, 2017.

La información obtenida con la aplicación de la Encuesta, se capturó en el programa informático de Excel, para lo cual se consideró construir una matriz de datos que se registró en columnas que representan las variables investigadas y en filas que representan la población estudiada.

\section{Aspectos sociodemográficos y regiones educativas}

La conformación de los Consejos Técnicos Regionales se realiza en apego al postulado de territorialidad del MGRH, en reconocimiento a la diversidad socio demográfica y escolar, existente en el estado.

El estado de Hidalgo al asumir el enfoque regional en su MGRH ha dado pasos relevantes en la conformación de colegios en las que se abre la posibilidad de reunión de las figuras de supervisión que conforman cada una de las regiones, y con ello establecer una condición para que los supervisores puedan dialogar, a partir de problemáticas compartidas, y tejer alianzas para buscar soluciones y tomar decisiones que respondan a la particularidad de su región. 


\section{Infraestructura}

El diagnóstico sobre infraestructura en las que funcionan los CTR no son las más adecuadas, de modo que se funciona es espacios inadecuados y con insuficiencia en la conectividad con internet.

Los CTR requieren ampliar y modernizar su infraestructura básica, lograr niveles adecuados de cobertura tecnológica y satisfacer con eficacia las necesidades de servicios de infraestructura de los espacios donde se desarrollen las sesiones colegiadas.

La infraestructura es necesaria, es importante; se trata de un elemento estratégico que debe ser tratado $y$ visto como tal, la infraestructura es clave, estratégica e indispensable para crear competitividad y continuidad operativa, es parte fundamental de su cadena de valor y su desatención puede traer un impacto negativo que se podrá prolongar hasta la desaparición de los colectivos estudiados.

\section{Población}

Los indicadores que fueron utilizados para el estudio de la población son: datos generales, formación profesional, experiencia laboral, área de influencia, así como las funciones que desarrollan, edad, género, años de servicio, años de servicio en la función, pertenencia indígena, condiciones laborales y regionales, entre otros.

En los CTR existe un predominio del género masculino, con edades entre los 40 a más de 70 años, con una antigüedad en el servicio predominante de más de 31 años y una antigüedad en la función para poco más de la mitad de ellos de entre 1 a 10 años.

El nivel de escolaridad que predomina entre los supervisores es el de Maestría, seguido de licenciatura, con condiciones contractuales la gran mayoría de Base, tiempo completo. Las diferencias entre los CTR se establecen por el nivel de marginalidad de los municipios, por la cantidad de población que atienden, y por la presencia de docentes que hablan una lengua indígena además del español.

\section{Estrategias de Operación de los CTR}

En el presente diagnóstico se han identificado las estrategias de operación considerando como referente en un primer momento los principios y postulados que plantea el MGRH, a partir de la sistematización y análisis de los datos derivados de la Encuesta se visualizan cuatro estrategias de operación que han hecho posible el funcionamiento de los CTR, ellas son: la colegialidad, Actualización del supervisor escolar, la cultura organizacional asociada a la lealtad y compromiso y la intervención estratégica. 
RECIE. Revista Electrónica Científica de Investigación Educativa Vol. 4, núm. 2, enero-diciembre 2019, pp. 1389-1395.

\section{Conclusiones}

Los CTR constituyen una estrategia para dar sentido al MGRH como instancia colegiada de supervisores y puente de comunicación entre la autoridad educativa estatal y la práctica de supervisión propiamente dicha, para promover la articulación básica y la atención de necesidades educativas en la región.

La población que conforma a los 30 CTR son: jefes de sector, supervisores generales, supervisores escolares y jefes de enseñanza, de los diferentes niveles y modalidades de Educación Básica en Hidalgo; 518 supervisores ofrecieron datos referenciales, el mayor predominio de supervisores son hombres (151 hombres por cada 100 mujeres), sus edades fluctúan entre 31 a más de 71 años. Los supervisores que se consideran indígenas son un $28.7 \%$ y de estos poco más del $50 \%$ habla náhuatl y el resto es hablante de HñäHñú.

La escolaridad de los supervisores se distribuye de la siguiente manera: $36.1 \%$ tiene estudios de maestría, un 31\% de licenciatura, el $10.4 \%$ tiene alguna especialidad, el $2.2 \%$ doctorado y el $9.9 \%$ Normal con Licenciatura, 9.9\% Normal Básica, y 0.5\% preparatoria o bachillerato; siendo el campo predominante de formación la Pedagogía.

La mayor parte ostenta una plaza base y tiempo completo, sin embargo, también existe personal que realiza la función supervisora en calidad de comisionados o cubriendo un interinato. Respecto a la antigüedad en la función, el mayor porcentaje tienen más de 31 años de antigüedad en el servicio educativo.

Los CTR han hecho posible la colegiabilidad entre sus integrantes quienes se reúnen con sus pares de manera sistemática y con regularidad, lo anterior ha permitido la continuidad y diversos grados de posicionamiento regional de estos consejos, asistir a ellos para la mayoría tiene motivos intrínsecos relacionados con la mejora de su desempeño profesional.

Por último, el diagnóstico ha hecho patente que para la mayoría de los supervisores es necesario sostener los CTR pero con cambios, encontrando ventajas para su fortalecimiento profesional, así como la necesidad de romper con las inercias establecidas, y revitalizar aquellos equipos que lo requieran, se trata entonces de conservar las fortalezas y reorientar las debilidades detectadas. 


\section{Referencias}

Arriaza Balmón, M. (2006). Guía práctica de análisis de datos. Consejería de Innovación, Ciencia y Empresa - Instituto de Investigación y Formación Agraria y Pesquera.

Hernández, R. (2010). Metodología de la investigación. México, McGraw-Hill. INEGI (2010). Índice de marginación municipal, Estado de Hidalgo. México: CONAPO e INEGI. Disponible en: http://poblacion.hidalgo.gob.mx/?p=1409

Olivé, A. (2011). Orientaciones para fortalecer las competencias profesionales de los equipos de supervisión en las Escuelas de Tiempo Completo, México, Dirección General de Desarrollo de la Gestión e Innovación Educativa de la Subsecretaría de Educación Básica de la Secretaría de Educación Pública.

Periódico Oficial del Estado de Hidalgo, (2011). Tomo CXLIV, Núm. 51 BIS. Pachuca, Hidalgo, México. Gobierno del estado Libre y Soberano de Hidalgo.

SEPH. (2014). Modelo de Gestión Regional Hidalgo, Secretaría de Educación Pública en el estado de Hidalgo- Hidalgo, México.

SEPH. (2015). Proyecto de investigación: El modelo de gestión y la función de la supervisión escolar en los Consejos Técnicos Regionales de Educación Básica del Estado de Hidalgo. Propuesta que se presenta para participar en la Convocatoria de Investigación en Educación Básica SEP/SEB-CONACYT, 2015. 
RECIE. Revista Electrónica Científica de Investigación Educativa Vol. 4, núm. 2, enero-diciembre 2019, pp. 1389-1395. 